\title{
Heterotic Standard Models from smooth Calabi-Yau Three-folds
}

\author{
Lara B. Anderson ${ }^{1}$, James Gray ${ }^{2}$, Andre Lukas ${ }^{* 3}$, Eran Palti ${ }^{4}$ \\ ${ }^{1}$ Center for the Fundamental Laws of Nature, Jefferson Laboratory \\ Harvard University, 17 Oxford Street, Cambridge, MA 02138, U.S.A. \\ ${ }^{2}$ Arnold-Sommerfeld-Center for Theoretical Physics, Department für Physik \\ Ludwig-Maximilians-Universität München, Theresienstraße 37, 80333 München, Germany. \\ ${ }^{3}$ Rudolf Peierls Centre for Theoretical Physics, University of Oxford, 1 Keble Road, Oxford, OXI \\ 3NP, U.K. \\ ${ }^{4}$ Centre de Physique Theorique, Ecole Polytechnique, CNRS, 91128 Palaiseau, France. \\ E-mail: lara@physics.harvard.edu, james.grayaphysik.uni-muenchen.de \\ lukas@physics.ox.ac.uk, Eran.Palti@cpht.polytechnique.fr
}

\begin{abstract}
We describe a new approach to heterotic Calabi-Yau model building, based on the systematic construction of vector bundles with Abelian structure groups. Starting with a relatively small number of Calabi-Yau manifolds, about 400 models with the exact matter spectrum of the supersymmetric standard model are found in this way. The additional, normally anomalous $U(1)$ symmetries which arise in these models place strong constraints on the allowed four-dimensional operators and can lead to interesting phenomenological consequences, some of which we discuss.
\end{abstract}

Proceedings of the Corfu Summer Institute 2011 School and Workshops on Elementary Particle Physics and Gravity

September 4-18, 2011

Corfu, Greece

\footnotetext{
*Speaker.
} 


\section{Introduction}

Compactification of the heterotic string on Calabi-Yau manifolds $[1,2]$ is the oldest approach to string phenomenology and remains one of the most promising avenues towards connection string theory with low-energy physics. However, despite considerable progress over the years, only a relatively small number of such models with a standard model spectrum [3]-[11] were known until recently, owing mainly to the mathematical difficulties of the construction. Any small number of string models with the right basic properties is likely to fail when confronted with more detailed phenomenological requirements, such as the correct spectrum of fermion masses. Constructing a true string standard model therefore depends on being able to construct quasi-realistic models systematically and in significant numbers.

Here, we would like to report on recent progress in this direction [12,13]. The main idea is to depart from traditional approaches to heterotic Calabi-Yau model building which have, for the most part, been using internal gauge fields with structure group $S U(n)$. Instead, we will consider gauge fields with an Abelian structure group. The resulting conceptual and technical simplifications, together with computational ground-work laid out in earlier papers [11, 14, 15], lead to the construction of several hundred heterotic standard models, that is models with the MSSM matter spectrum, one or more pairs of Higgs doublets and no exotics charged under the standard model group.

To explain this in more detail we have to recall the data required for a Calabi-Yau compactification of the $E_{8} \times E_{8}$ heterotic string. In additional to a Calabi-Yau three manifold $X$, we need "observable" and "hidden" vector bundles $V$ and $\tilde{V}$ on $X$, both supersymmetric and with a structure group which is a sub-group of $E_{8}$, and a holomorphic curve $C \subset X$ which is wrapped by five-branes. These various objects are subject to the anomaly constraint

$$
\operatorname{ch}_{2}(V)+\operatorname{ch}_{2}(\tilde{V})-\operatorname{ch}_{2}(T X)=[C],
$$

where the square brackets indicate the homology class of the curve $C$. Here, we will only construct the Calabi-Yau three-fold $X$ and the observable bundle $V$ explicitly. However, we will ensure that a supersymmetric, anomaly-free completion of the model always exists. To do this we require that $\operatorname{ch}_{2}(V)-\operatorname{ch}_{2}(T X)$ is such that the anomaly condition (1.1) can be satisfied by a suitable choice of holomorphic curve $C$. (In more technical terms, we require $\operatorname{ch}_{2}(V)-\operatorname{ch}_{2}(T X)$ to be an element of the Mori cone of $X$.)

A typical choice for the structure group of the bundle $V$ has been the group $S U(5)$ which leads to a low-energy $S U(5)$ GUT theory. Here, we use instead bundles $V$ with the Abelian structure group $S\left(U(1)^{5}\right) \subset S U(5)$. Such bundles are easily constructed as a sum of five line bundles

$$
V=\bigoplus_{a=1}^{5} L_{a}
$$

satisfying $c_{1}(V)=\sum_{a} c_{1}\left(L_{a}\right)=0$. Line bundles are classified by their first Chern class and, hence, such line bundle sums can be constructed systematically. Moreover, properties of $V$ required to compute properties of the associated low-energy theory are much easier accessible for line bundles.

An interesting general feature of such models is that the structure group $S\left(U(1)^{5}\right)$ is selfcommuting and, hence, also appears as a low-energy gauge symmetry. The full low-energy gauge 
group, before Wilson-line breaking, for such line bundle models is therefore $S U(5) \times S\left(U(1)^{5}\right)$. Usually, the additional $U(1)$ symmetries are Green-Schwarz anomalous so that the associated vector bosons are super-massive and do not cause a phenomenological problem. However, the $U(1)$ symmetries still restrict the set of allowed operators in the four-dimensional theory. This leads to a range of interesting phenomenological possibilities, for example in relation to proton stability, fermion masses, $\mu$-problem etc. In order to discuss these issues, we should first introduce heterotic line bundle models in more detail.

\section{Heterotic line bundle models}

To construct heterotic line bundle models we start with a Calabi-Yau three-fold $X$, with $h^{1,1}(X)$ Kahler parameters, $t^{i}$, so that the Kahler form can be written as $J=t^{i} \omega_{i}$, relative to some basis of harmonic two-forms $\left\{\omega_{i}\right\}$. We will also require the existence of a freely-acting symmetry $\Gamma$ of $X$, in order to be able to form the non simply-connected quotient $\hat{X}=X / \Gamma$. On this quotient, Wilson lines can be introduced in a subsequent step of the construction which can break the $S U(5)$ GUT group to the standard model.

As mentioned above, line bundles $L$ are characterized by their first Chern class $c_{1}(L)=k^{i} \omega_{i}$ and, hence, by a set of $h^{1,1}(X)$ integers $\mathbf{k}=\left(k^{i}\right)$. We will write a line bundle with this Chern class as $\mathscr{O}_{X}(\mathbf{k})$. A line bundle sum of the required form (1.2) then consists of five line bundles $L_{a}=\mathscr{O}_{X}\left(\mathbf{k}_{a}\right)$ and is, hence, simply given by an integer matrix $\left(k_{a}^{i}\right)$ of size $h^{1,1}(X) \times 5$, satisfying

$$
\sum_{a=1}^{5} k_{a}^{i}=0
$$

to ensure that $c_{1}(V)=0$. Such line bundle sums are supersymmetric if all their slopes vanish simultaneously, that is, if the equations

$$
d_{i j l} t^{i} t^{j} k_{a}^{l}=0
$$

(where $d_{i j k}$ are the triple intersection numbers of the Calabi-Yau manifold $X$ ) have a common solution $t_{i}$ in Kahler moduli space. A necessary condition for the existence of such a solutions is

$$
\operatorname{rank}\left(k_{a}^{i}\right)<h^{1,1}(X) .
$$

Since we are considering rank five bundles this constraint means that model building options are severely restricted for Calabi-Yau manifolds with a small number of Kahler paramenters, $h^{1,1}(X)<$ 5 .

The heterotic string on $X$ with such a line bundle sum $V$ leads to a four-dimensional GUT theory with gauge group $S U(5) \times S\left(U(1)^{5}\right)$ and matter multiplets

$$
\mathbf{1 0} \mathbf{e}_{a}, \overline{\mathbf{1 0}}_{-\mathbf{e}_{a}}, \overline{\mathbf{5}}_{\mathbf{e}_{a}+\mathbf{e}_{b}}, \mathbf{5}_{-\mathbf{e}_{a}-\mathbf{e}_{b}}, \mathbf{1}_{\mathbf{e}_{a}-\mathbf{e}_{b}} .
$$

Here, $\mathbf{e}_{a}$ denotes the $a^{\text {th }}$ standard unit vector in five dimensions and the subscripts indicate the charges of the respective $S U(5)$ multiplet under the $S\left(U(1)^{5}\right)$ symmetry. In general, we describe irreducible $S\left(U(1)^{5}\right)$ representations by integer vectors $\mathbf{q}=\left(q_{1}, \ldots, q_{5}\right)$. Due to the special unitarity 
condition, two such vectors, $\mathbf{q}$ and $\tilde{\mathbf{q}}$, describe the same representation and have to be identified if $\mathbf{q}-\tilde{\mathbf{q}} \in \mathbb{Z}(1,1,1,1,1)$.

The additional $U(1)$ symmetries in $S\left(U(1)^{5}\right)$ are normally Green-Schwarz anomalous and, hence, the associated vector bosons are super-massive. However, in special cases, some of the $U(1)$ symmetries may be massless and, hence, anomaly-free. The number of such symmetries can be easily computed from

$$
\text { (Number of massless } U(1) \text { symmetries) }=4-\operatorname{rank}\left(k_{a}^{i}\right) .
$$

Comparison with Eq. (2.3) shows that models on Calabi-Yau manifolds with $h^{1,1}(X)<5$ necessarily have at least one massless $U(1)$ symmetry. For $h^{1,1}(X) \geq 5$ massless $U(1)$ symmetries can arise for special, non-generic choices of the line bundles $k_{a}^{i}$. In any case, the appearance of such massless $U(1)$ symmetries is not necessarily a phenomenological disaster. We note from the spectrum (2.4) that the GUT singlets do, in fact, carry charges under $S\left(U(1)^{5}\right)$. Hence, turning on VEV s for these singlets (which corresponds to moving to a locus in the bundle moduli where the bundle becomes non-Abelian), these massless $U(1)$ symmetries can easily be broken spontaneously.

Note that each GUT multiplet comes with specific $S\left(U(1)^{5}\right)$ charges which significantly restrict the allowed operators in the four-dimensional theory. For example, the standard $S U(5)$ Yukawa couplings

$$
\mathbf{5}_{-\mathbf{e}_{a}-\mathbf{e}_{b}}^{\bar{H}} \mathbf{1 0} \mathbf{e}_{c} \mathbf{1 0} \mathbf{e}_{d}, \quad \overline{\mathbf{5}}_{\mathbf{e}_{a}+\mathbf{e}_{b}}^{H} \overline{\mathbf{s}}_{\mathbf{e}_{c}+\mathbf{e}_{d}} \mathbf{1 0} \mathbf{e}_{f}
$$

are only allowed if $\mathbf{e}_{a}+\mathbf{e}_{b}=\mathbf{e}_{c}+\mathbf{e}_{d}$ and $\mathbf{e}_{a}+\mathbf{e}_{b}+\mathbf{e}_{c}+\mathbf{e}_{d}+\mathbf{e}_{f}=(1,1,1,1,1)$, respectively.

The number of each type of GUT multiplet can be computed from line bundle cohomology as summarized in Table 1. As we will discuss later, for certain classes of Calabi-Yau manifolds the

\begin{tabular}{|l|c|c|c|}
\hline multiplet & $S\left(U(1)^{5}\right)$ charge & associated line bundle $L$ & contained in \\
\hline \hline $\mathbf{1 0}_{\mathbf{e}_{a}}$ & $\mathbf{e}_{a}$ & $L_{a}$ & $V$ \\
\hline$\overline{\mathbf{1 0}}_{-\mathbf{e}_{a}}$ & $-\mathbf{e}_{a}$ & $L_{a}^{*}$ & $V^{*}$ \\
\hline$\overline{\mathbf{5}}_{\mathbf{e}_{a}+\mathbf{e}_{b}}$ & $\mathbf{e}_{a}+\mathbf{e}_{b}$ & $L_{a} \otimes L_{b}$ & $\wedge^{2} V$ \\
\hline $\mathbf{5}_{-\mathbf{e}_{a}-\mathbf{e}_{b}}$ & $-\mathbf{e}_{a}-\mathbf{e}_{b}$ & $L_{a}^{*} \otimes L_{b}^{*}$ & $\wedge^{2} V^{*}$ \\
\hline $\mathbf{1}_{\mathbf{e}_{a}-\mathbf{e}_{b}}$ & $\mathbf{e}_{a}-\mathbf{e}_{b}$ & $L_{a} \otimes L_{b}^{*}$ & $V \otimes V^{*}$ \\
$\mathbf{1}_{-\mathbf{e}_{a}+\mathbf{e}_{b}}$ & $-\mathbf{e}_{a}+\mathbf{e}_{b}$ & $L_{a}^{*} \otimes L_{b}$ & \\
\hline
\end{tabular}

Table 1: Multiplet content, charges and associated line bundles of the $S U(5) \times S\left(U(1)^{5}\right)$ GUT theory. The indices $a, b, \ldots$ are in the range $1, \ldots, 5$ and $\mathbf{e}_{a}$ denotes the standard five-dimensional unit vector in the $a^{\text {th }}$ direction. The number of each type of multiplet is obtained from the first cohomology, $H^{1}(X, L)$, of the associated line bundle $L$.

techniques required to calculate these line bundle cohomologies are available, so that the complete GUT spectrum, including the values of the $S\left(U(1)^{5}\right)$ charges can be computed explicitly. To obtain a standard model spectrum we require the absence of mirror families. At the present GUT stage this means we should not allow any $\overline{\mathbf{1 0}}$ multiplets. Hence, we will only use line bundles $L_{a}$ satisfying

$$
h^{2}\left(X, L_{a}\right)=h^{1}\left(X, L_{a}^{*}\right) \stackrel{!}{=} 0 .
$$

Further, in order to produce three chiral families downstairs, we demand that

$$
h^{1}(X, V)=\sum_{a} h^{1}\left(X, L_{a}\right) \stackrel{!}{=} 3|\Gamma|, \quad h^{1}\left(X, \wedge^{2} V\right)-h^{1}\left(X, \wedge^{2} V^{*}\right) \stackrel{!}{=} 3|\Gamma|
$$




\begin{tabular}{|l|l|l|l|}
\hline$S U(5)$ repr. & $G_{\text {SM }}$ repr. & name & cohomology \\
\hline \hline $\mathbf{1 0}_{\mathbf{e}_{a}}$ & $(\mathbf{3}, \mathbf{2})_{1}$ & $Q_{a}$ & $h^{1}\left(X, L_{a}, \chi_{a} \otimes W^{*} \otimes \tilde{W}^{*}\right)$ \\
& $(\overline{\mathbf{3}}, \mathbf{1})_{-4}$ & $u_{a}$ & $h^{1}\left(X, L_{a}, \chi_{a} \otimes W^{*} \otimes W^{*}\right)$ \\
& $(\mathbf{1}, \mathbf{1})_{6}$ & $e_{a}$ & $h^{1}\left(X, L_{a}, \chi_{a} \otimes \tilde{W}^{*} \otimes \tilde{W}^{*}\right)$ \\
\hline$\overline{\mathbf{5}}_{\mathbf{e}_{a}+\mathbf{e}_{b}}$ & $(\overline{\mathbf{3}}, \mathbf{1})_{2}$ & $d_{a, b}, T_{a, b}$ & $h^{1}\left(L_{a} \otimes L_{b}, \chi_{a} \otimes \chi_{b} \otimes W\right)$ \\
& $(\mathbf{1}, \mathbf{2})_{-3}$ & $L_{a, b}, H_{a, b}$ & $h^{1}\left(L_{a} \otimes L_{b}, \chi_{a} \otimes \chi_{b} \otimes \tilde{W}\right)$ \\
\hline $\mathbf{5}_{-\mathbf{e}_{a}-\mathbf{e}_{b}}$ & $(\mathbf{3}, \mathbf{1})_{-2}$ & $\bar{T}_{a, b}$ & $h^{2}\left(L_{a} \otimes L_{b}, \chi_{a} \otimes \chi_{b} \otimes W\right)$ \\
& $(\mathbf{1}, \mathbf{2})_{3}$ & $\bar{H}_{a, b}$ & $h^{2}\left(L_{a} \otimes L_{b}, \chi_{a} \otimes \chi_{b} \otimes W\right)$ \\
\hline $\mathbf{1}_{\mathbf{e}_{a}-\mathbf{e}_{b}}$ & $(\mathbf{1}, \mathbf{1})_{0}$ & $S_{a, b}$ & $h^{1}\left(L_{a} \otimes L_{b}^{*}, \chi_{a} \otimes \chi_{b}^{*}\right)$ \\
\hline
\end{tabular}

Table 2: Cohomologies which compute the downstairs spectrum. The Wilson line is characterized by the two representations $W$ and $\tilde{W}$ of the freely-acting Abelian symmetry $\Gamma$ and $\chi_{a}^{*}$ are the characters of the line bundles $L_{a}$. The notation $h^{1}(X, L, R)$ in the last column denotes the dimension of the subspace of $H^{1}(X, L)$ which transforms under the representation $R$ of $\Gamma$.

where $|\Gamma|$ is the order of the discrete symmetry group $\Gamma$. Note that we do not require the absence of 5 multiplets. Indeed, we need at least one such multiplet in order to be able to obtain a pair of Higgs doublets, so we require that

$$
h^{1}\left(X, \wedge^{2} V^{*}\right) \stackrel{!}{>} 0
$$

To break the GUT models to the standard model, we need to divide by the freely-acting symmetry $\Gamma$ and introduce Wilson lines. In addition, the bundle $V$ needs to descend to a bundle $\hat{V}$ on the quotient manifold $\hat{X}=X / \Gamma$. The corresponding mathematical notion is that the bundle $V$ be equivariant under $\Gamma$, a condition which can be checked explicitly for line bundle sums, at least on certain Calabi-Yau manifolds. The complete downstairs bundle is then $\hat{V} \oplus \mathscr{W}$, where $\mathscr{W}$ is a flat bundle which corresponds to the Wilson line. The number of downstairs multiplets is given in terms of the cohomology of $\hat{V} \oplus \mathscr{W}$, but can, more easily, be computed by considering the $\Gamma$ representation properties of the upstairs GUT spectrum.

To discuss this more explicitly, we assume that $\Gamma=\otimes_{r} \mathbb{Z}_{n_{r}}$ is an Abelian symmetry and that the bundle is $\Gamma$ equivariant due to every line bundle $L_{a}$ being equivariant individually. In this case, the equivariant structure on $V$ can be described by an arbitrary choice of five $\Gamma$-representations ("characters") $\chi_{a}^{*}$. Further, the Wilson line, embedded in the hypercharge direction in $S U(5)$, is characterized by two $\Gamma$-representations, $W$ and $\tilde{W}$, subject to the condition $W^{3} \otimes \tilde{W}^{2}=1$. Within this set-up, the number of downstairs multiplets can be computed by working out the $\Gamma$ representation content of upstairs cohomologies, as indicated in Table 2. For certain classes of Calabi-Yau manifolds this can be carried out explicitly, as we will discuss in the following section.

Note from Table 2 that each standard model multiplet will inherit the $S\left(U(1)^{5}\right)$ representation of its underlying GUT multiplet. The pattern of operators allowed or forbidden under $S\left(U\left(1^{5}\right)\right.$ is, therefore, consistent with an $S U(5)$ grand unified symmetry, although the actual values of coupling constants can, of course, break the GUT symmetry. For example, the tau and bottom Yukawa couplings are either both forbidden or allowed by $S\left(U(1)^{5}\right)$, however, in the latter case, their values are not necessarily identical. 
In order to obtain a spectrum without any exotics we should impose further phenomenological constraints which eliminate all Higgs triplets, $T, \bar{T}$ and ensure we are left with at least one pair of Higgs doublets. From Table 2 this means we should require that

$$
\begin{aligned}
& h^{2}\left(L_{a} \otimes L_{b}, \chi_{a} \otimes \chi_{b} \otimes W\right) \stackrel{!}{=} 0 \text { for all } a, b \\
& h^{2}\left(L_{a} \otimes L_{b}, \chi_{a} \otimes \chi_{b} \otimes \tilde{W}\right) \stackrel{!}{>} 0 \text { for at least one } a, b .
\end{aligned}
$$

If these and the previous phenomenological constraints are satisfied, the resulting four-dimensional theory has $N=1$ supersymmetry, a standard model gauge group (times $S\left(U(1)^{5}\right)$, normally anomalous), three families of matter, one or more pair of Higgs doublets, a set of standard model singlets which, however, are charged under $S\left(U(1)^{5}\right)$ and no exotics charged under the standard model group of any kind. In other words, we have an NMSSM-like model with a number of additional singlet field. Operators in this theory will be restricted by $S\left(U(1)^{5}\right)$, in a way which is consistent with an $S U(5)$ GUT symmetry. As we will now see, all of the above mathematical and physical requirements can indeed be met for certain classes of Calabi-Yau manifolds.

\section{Line bundle standard models on CICYs}

Complete intersection Calabi-Yau manifolds (CICYs) are defined as the common zero locus of polynomials in products of projective spaces. They are arguably the simplest Calabi-Yau manifolds (with the quintic in $\mathbb{P}^{4}$ as their most prominent representative) and, according to the classification of Ref. [16], there are about 8000 such spaces. All required quantities, such as second Chern class, Euler number, Hodge numbers and intersection numbers have been explicitly computed for these manifolds ${ }^{1}$. The range of allowed Kahler parameters (the "Kahler cone") for favourable CICYs is $t^{i}>0$, for all $i$. Crucially, more recently, freely-acting symmetries for these manifolds have been classified [17] and methods to compute line bundle cohomology on CICYs have been developed $[11,15]$. Hence, all the required technical ingredients discussed in the previous section are available for CICYs and, currently, they constitute the only class of Calabi-Yau manifolds for which this is the case.

As we have discussed, the complication of our models increases with increasing number, $h^{1,1}(X)$, of Kahler parameters, since each line bundle is characterized by as many integers. For this reason, we have searched through the list of CICYs starting with a small number of Kahler parameters and gradually increasing $h^{1,1}(X)$. In this way, we have covered all (favourable) CICYs with $h^{1,1}(X) \leq 5$, in total about 60 manifolds. For each such manifold, we have generated all line bundle sums, $\left(k_{a}^{i}\right)$, with entries in a given range, and have checked the various mathematical and physical conditions explained in the previous section. On manifolds with $h^{1,1}(X)=1$ the bundle supersymmetry constraints (2.2) cannot be satisfied, so such manifolds (which include the quintic) are ruled out for line bundle models. For the manifolds with $h^{1,1}(X)=2\left(h^{1,1}(X)=3\right)$, we have covered the range $-10 \leq k_{a}^{i} \leq 10\left(-3 \leq k_{a}^{i} \leq 3\right)$. In either case, no examples consistent with all requirements have been found, that is, there are no line bundle standard model on such CICYs. Intuitively, the reason for this is the condition (2.3) which is necessary for supersymmetry

\footnotetext{
${ }^{1}$ The complete set of intersection numbers is currently only available for "favourable" CICYs, that is CICYs for which the entire second cohomology descends from the ambient space. Here, we will focus on such favourable CICYs.
} 
of the bundle. It constitutes a strong constraint for small $h^{1,1}(X)$ and, combined with the physical conditions on the spectrum, it rules out all models for $h^{1,1}(X)=2,3$.

For the $h^{1,1}(X)=4\left(h^{1,1}(X)=5\right)$ manifolds we have covered the range $-3 \leq k_{a}^{i} \leq 3(-2 \leq$ $\left.k_{a}^{i} \leq 2\right)$ and we have found a substantial number of viable models. At the level of the underlying GUT theories, there are about 200 models with the right properties. It turns out, they are all associated to discrete symmetries $\Gamma=\mathbb{Z}_{2}$ or $\Gamma=\mathbb{Z}_{2} \times \mathbb{Z}_{2}$. Each of these models is divided by $\Gamma$ and all possible choices, $\chi_{a}^{*}$, for the bundle equivariant structure and the Wilson line, $W$ and $\tilde{W}$, are explored. This leads to between $\mathscr{O}(100)$ to $\mathscr{O}(1000)$ choices for each of the 200 GUT models. Not all of these choices are viable, for example, for some the resulting spectrum contains Higgs triplets. Keeping only the models with a viable spectrum and only one representative per spectrum obtained we end up with 399 standard models.

Each of these 399 models has a standard model gauge group times $S\left(U(1)^{5}\right.$, precisely three standard model families, one, two or three pairs of Higgs doublets, a number of standard model singlets (bundle moduli) and no exotics charged under the standard model group. For 233 of the models all four additional $U(1)$ symmetries are Green-Schwarz anomalous and, hence, massive, the remaining models have one or occasionally two massless $U(1)$ symmetries. The numbers of models with one, two and three pairs of Higgs doublets are $(259,72,63)$.

The matter field content for each of these models consists of the three families $\left(\mathbf{1 0}_{a_{p}}\right)=$ $\left(Q_{a_{p}}, u_{a_{p}}, e_{a_{p}}\right)$ and $\left(\overline{\mathbf{5}}_{b_{p}, c_{p}}\right)=\left(d_{b_{p}, c_{p}}, L_{b_{p}, c_{p}}\right)$, where $p, q, \ldots=1,2,3$ are family indices, and one or more pairs, $H_{g, h}, \bar{H}_{\bar{g}, \bar{h}}$ of Higgs doublets and singlets $S_{d_{\alpha}, f_{\alpha}}$. The notation is such that the $S\left(U(1)^{5}\right.$ charges, $\mathbf{Q}$, for these multiplets are given by

$$
\begin{aligned}
& \mathbf{Q}\left(\mathbf{1 0}_{a_{p}}\right)=\mathbf{e}_{a_{p}} \quad, \mathbf{Q}\left(\overline{\mathbf{5}}_{b_{p}, c_{p}}\right)=\mathbf{e}_{b_{p}}+\mathbf{e}_{c_{p}} \\
& \mathbf{Q}\left(H_{g, h}\right)=\mathbf{e}_{h}+\mathbf{e}_{g} \quad, \mathbf{Q}\left(\bar{H}_{\bar{g}, \bar{h}}\right)=-\mathbf{e}_{\bar{g}}-\mathbf{e}_{\bar{h}} \\
& \mathbf{Q}\left(S_{d_{\alpha}, f_{\alpha}}\right)=\mathbf{e}_{d_{\alpha}}-\mathbf{e}_{f_{\alpha}} .
\end{aligned}
$$

The indices $a_{p}, b_{p}, \ldots=1, \ldots, 5$ specify the $S\left(U(1)^{5}\right)$ charges for the various multiplets and are of course fixed for each of our explicit models. For a given model, the pattern of these charges restricts the allowed operators in the four-dimensional theory which has potential implications for a whole range of phenomenological issues. For example, the $S\left(U(1)^{5}\right.$ symmetry may forbid dimension four and five proton decay violating operators. This indeed happens for 195 out of the 399 models. It is important to note that the singlet fields, $S$, also carry $S\left(U(1)^{5}\right)$ charges so that their couplings to standard model fields are constrained. For example, this means that the $S\left(U(1)^{5}\right)$ symmetry, together with (small) singlet VEVs can form the basis of a Froggatt-Nielsen type appoach towards obtaining the correct fermion masses. An elementary phenomenological requirement is that the top Yukawa coupling is allowed by the $S\left(U(1)^{5}\right)$ symmetry. This turns out to be the case for 45 of our models. If we combine the requirements of precisely one pair of Higgs doublets, a stable proton, no massless $U(1)$ symmetry and an allowed top Yukawa coupling, we find 13 corresponding models.

A searchable database of all 399 models, suitable for a more detailed phenomenological analysis, can be obtained from the webpage [18]. In addition to the underlying Calabi-Yau and bundle data this database also includes lists of the four-dimensional operators allowed by $S\left(U(1)^{5}\right)$. 


\section{An example}

To be more specific, we would now like to present an explicit example from the database [18]. The underlying CICY manifold for this example if defined by the common zero locus of two polynomials with multi-degrees $(0,1,1,1,1)$ and $(2,1,1,1,1)$ in the ambient space $\left(\mathbb{P}^{1}\right)^{\times 5}$. The two generators of the freely-acting symmetry $\Gamma=\mathbb{Z}_{2} \times \mathbb{Z}_{2}$ act as

$$
\left(\begin{array}{rr}
1 & 0 \\
0 & -1
\end{array}\right), \quad\left(\begin{array}{ll}
0 & 1 \\
1 & 0
\end{array}\right)
$$

on the two homogeneous coordinates of each ambient space $\mathbb{P}^{1}$ factor. This CICYs has five Kahler parameters, $h^{1,1}(X)=5$, so that a line bundle is specified by five integers. The relevant line bundle sum for our example is given by

$$
\left(k_{a}^{i}\right)=\left(\begin{array}{rrrrr}
1 & 1 & 0 & 0 & -2 \\
-1 & -1 & 1 & 0 & 1 \\
0 & -1 & 0 & 1 & 0 \\
0 & 0 & -2 & 1 & 1 \\
0 & 1 & 1 & -2 & 0
\end{array}\right) .
$$

Note that the columns of this matrix, the vectors $\mathbf{k}_{a}$, define the five line bundles $L_{a}=\mathscr{O}_{X}\left(\mathbf{k}_{a}\right)$. The Wilson line is chosen as $W=(0,0), \tilde{W}=(0,1)$ and the equivariant structure as $\left(\chi_{a}^{*}\right)=$ $((0,0),(0,0),(0,0),(0,1),(0,0))$, where the integer pairs denote $\mathbb{Z}_{2} \times \mathbb{Z}_{2}$ charges. Then, the resulting matter spectrum is

$$
\left(\mathbf{1 0}_{3}, \mathbf{1 0}_{4}, \mathbf{1 0}_{5}, \overline{\mathbf{5}}_{1,3}, \overline{\mathbf{5}}_{1,4}, \overline{\mathbf{5}}_{4,5}, H_{3,4}, \bar{H}_{3,4}, S_{4,1}, 3 S_{5,1}, 3 S_{4,2}, 3 S_{5,2}, 2 S_{3,4}, 2 S_{4,3}, 3 S_{3,5}\right) .
$$

We recall, that the subscripts specify the $S\left(U(1)^{5}\right)$ charges. For example, the notation $\mathbf{1 0}_{3}$ indicates that this multiplet has charge $\mathbf{Q}\left(\mathbf{1 0}_{3}\right)=\mathbf{e}_{3}$.

It is easy to see that the up-type Yukawa coupling $\bar{H}_{3,4} \mathbf{1 0}_{3} \mathbf{1 0}_{4}$ is allowed, so that this model can accommodate on order one top Yukawa coupling, while all down-type Yukawa couplings, $H \overline{\mathbf{5}} \mathbf{1 0}$, are forbidden. In fact, the down-type Yukawa couplings remain zero even if we allow singlet VEV insertions of the singlet fields available in the spectrum (4.3). This means that non-perturbative effects are required in this model to generate the down-type Yukawa couplings. It can also be checked from the charges in (4.3) that all dimension four and five proton-decay violating operators of the form $\mathbf{1 0 5} \overline{5}$ and $\mathbf{5} \mathbf{1 0 1 0 1 0}$, are forbidden even when singlet VEV insertions are allowed.

\section{Conclusion}

We have seen that heterotic line bundle models constitute an interesting class of string models which allows for the construction of large numbers of quasi-realistic models. In addition, thanks to the additional $S\left(U(1)^{5}\right)$ symmetry in those models, a number of relevant phenomenological issues can be discussed without the need for a full-scale calculation of the four-dimensional coupling constants from string theory.

A relatively modest scan, based on 60 Calabi-Yau manifolds and line bundles with entries $k_{a}^{i}$ in a fairly restricted range, has already led to about 400 standard models which are accessible from a 
database [18]. Significant extensions of this scan, both to a larger number of Calabi-Yau manifolds and a larger class of line bundles, are feasible and currently underway. We anticipate that this will lead to many thousands of standard models. A large set of quasi-realistic string models is required in order to search for a true string standard model, that is, a model which reproduces all known features of low-energy physics. We hope that a comprehensive scan for quasi-realistic line bundle standard models will provide such a starting point and will ultimately lead to the construction of a fully realistic string standard model.

\section{Acknowledgments}

L. A. is supported by the Fundamental Laws Initiative of the Center for the Fundamental Laws of Nature, Harvard University. J. G. would like to acknowledge support by the NSF-Microsoft grant NSF/CCF-1048082. A. L. is supported in part by the EC 6th Framework Programme MRTN-CT2004-503369 and EPSRC network grant EP/I02784X/1. The work of E. P. is supported by a Marie Curie Intra European Fellowship within the 7th European Community Framework Programme.

\section{References}

[1] P. Candelas, G. T. Horowitz, A. Strominger, E. Witten, "Vacuum Configurations for Superstrings," Nucl. Phys. B258 (1985) 46-74.

[2] M. B. Green, J. H. Schwarz and E. Witten, "Superstring theory. Vol. 2: Loop amplitudes, anomalies and phenomenology," Cambridge, Uk: Univ. Pr. ( 1987) 596 P. ( Cambridge Monographs On Mathematical Physics)

[3] B. R. Greene, K. H. Kirklin, P. J. Miron and G. G. Ross, "A Three Generation Superstring Model. 1. Compactification and Discrete Symmetries,” Nucl. Phys. B 278 (1986) 667.

[4] B. R. Greene, K. H. Kirklin, P. J. Miron and G. G. Ross, “A Three Generation Superstring Model. 2. Symmetry Breaking and the Low-Energy Theory,” Nucl. Phys. B 292 (1987) 606.

[5] V. Braun, P. Candelas, R. Davies and R. Donagi, "The MSSM Spectrum from (0,2)-Deformations of the Heterotic Standard Embedding,” arXiv:1112.1097 [hep-th].

[6] V. Braun, Y. H. He, B. A. Ovrut and T. Pantev, “A Heterotic standard model,” Phys. Lett. B 618, 252 (2005) [arXiv:hep-th/0501070].

[7] V. Braun, Y. H. He, B. A. Ovrut and T. Pantev, "A Standard model from the E(8) x E(8) heterotic superstring,” JHEP 0506, 039 (2005) [arXiv:hep-th/0502155].

[8] V. Braun, Y. H. He, B. A. Ovrut and T. Pantev, "Vector bundle extensions, sheaf cohomology, and the heterotic standard model," Adv. Theor. Math. Phys. 10, 4 (2006) [arXiv:hep-th/0505041].

[9] V. Bouchard and R. Donagi, “An SU(5) heterotic standard model,” Phys. Lett. B 633, 783 (2006) [arXiv:hep-th/0512149].

[10] V. Braun, Y. H. He, B. A. Ovrut and T. Pantev, “The Exact MSSM spectrum from string theory," JHEP 0605, 043 (2006) [arXiv:hep-th/0512177].

[11] L. B. Anderson, J. Gray, Y. -H. He and A. Lukas, "Exploring Positive Monad Bundles And A New Heterotic Standard Model,” JHEP 1002 (2010) 054 [arXiv:0911.1569 [hep-th]]. 
[12] L. B. Anderson, J. Gray, A. Lukas and E. Palti, "Heterotic Line Bundle Standard Models," arXiv:1202.1757 [hep-th].

[13] L. B. Anderson, J. Gray, A. Lukas and E. Palti, "Two Hundred Heterotic Standard Models on Smooth Calabi-Yau Threefolds,” Phys. Rev. D 84 (2011) 106005 [arXiv:1106.4804 [hep-th]].

[14] L. B. Anderson, Y. -H. He and A. Lukas, "Monad Bundles in Heterotic String Compactifications," JHEP 0807 (2008) 104 [arXiv:0805.2875 [hep-th]].

[15] L. B. Anderson, Y. -H. He and A. Lukas, "Heterotic Compactification, An Algorithmic Approach," JHEP 0707 (2007) 049 [hep-th/0702210 [HEP-TH]].

[16] P. Candelas, A. M. Dale, C. A. Lutken and R. Schimmrigk, "Complete Intersection Calabi-Yau Manifolds," Nucl. Phys. B 298 (1988) 493.

[17] V. Braun, “On Free Quotients of Complete Intersection Calabi-Yau Manifolds,” JHEP 1104, 005 (2011) [arXiv:1003.3235 [hep-th]].

[18] The database of 400 heterotic line bundle standard models can be accessed at http://www-thphys.physics.ox.ac.uk/projects/CalabiYau/linebundlemodels/index.html. 